\title{
Diffuse Interstellar Bands: Families and Correlations
}

\author{
J. Krełowski
}

Toruń Centre for Astronomy, Nicolaus Copernicus University, Faculty of Physics, Astronomy and Applied Informatics, Grudziądzka 5 Pl-87-100 Toruń, Poland

email: jacek@astri.uni.torun.pl

\begin{abstract}
The term "families of diffuse bands" (DIBs) appeared in 1986/87 when my collaborators: Gordon A.H. Walker, Bengt E. Westerlund and I found that the strength ratio of the major DIBs 5780 and 5797 is heavily variable. We proved that at the same E(B-V) the DIB intensities may vary by as much as a factor of three or more. A similar result was published by Karl Josafatsson and Ted Snow soon after. A decade later, we proved (with Chris Sneden) that certain DIB strength ratios seem to be related to intensities of the known features of simple molecular species; this led to the introduction of the so called $\sigma$ and $\zeta$ type interstellar clouds. The former are characterized by very weak molecular features (but broad DIBs - very strong) while the latter by rather strong bands of simple radicals and weak broad DIBs. Currently we face a bunch of questions: are the DIB intensities related to those of certain molecular species, e.g. $C_{2}$ as suggested by Lew Hobbs' and Ted Snow's group? Do the DIB profiles, found to be complex by Peter Sarre, depend on e.g. the rotational temperatures of simple, linear carbon species? Do the DIB profiles depend on the irradiation of interstellar clouds by nearby stars? The relative DIB strengths as well as those of the simple radicals seem to be related to the shapes of interstellar extinction curves. We thus face three players in the interstellar translucent clouds: dust particles, simple radicals and the DIB carriers. Apparently, their mutual relations depend on local physical parameters of intervening clouds; these relations are not clear yet.
\end{abstract}

Keywords. ISM: lines and bands, ISM: molecules, ISM: extinction

\section{Introduction}

Diffuse interstellar bands appeared in the literature in 1922, i.e. more than 90 years ago (Heger 1922) as described in a nice, recent paper by McCall and Griffin (2013). However, the term "diffuse interstellar bands" was introduced later by P.W. Merrill (1938) who, together with the collaborators from the Mount Wilson Observatory, was the first to investigate these puzzling features systematically. At the time only six DIBs were known, those whose current identifications are: 5780, 5797, 6203, 6269, 6284 and 6614 .

The bands are considered to be interstellar because:

- they are stationary features in the spectra of spectroscopic binaries

- they seem to be correlated with other, identified interstellar features

- they are not observed in the spectra of unreddened stars

- their intensities grow with $\mathrm{E}(\mathrm{B}-\mathrm{V})$.

They bear the name "diffuse" because their profiles are broader than those of the well known atomic/molecular lines even though the latter are much stronger.

The number of known diffuse interstellar bands (DIBs) initially grew very slowly. By the end of the 1960s there were 9 listed DIBs. It seemed thus natural that they are carried by the same substance. The latter remained, however, unknown. In the early 1970s George Herbig was the first to use a computer to average tracings of $8-10$ individual 
spectra of every reddened star he observed. The result was the famous publication (Herbig 1975) which listed 39 DIBs plus a couple of suspects. This result created several doubts concerning the common origin of all DIBs and the hypothesis that they are carried by dust grains - then popular. Figure 3 from the above paper created a kind of "standard" picture of diffuse bands seen in the spectra of reddened stars for several years.

With the advent of solid state receivers, astrophysical spectra acquired a property hardly imaginable before: a high signal-to-noise (SNR) ratio available even in single exposures. Moreover, when digitally recorded they can easily be averaged. This made it possible to trace the profiles of weak DIBs or strong ones in slightly reddened spectra. In addition, the intensities of observed features could be measured with impressive precision. This was an important breakthrough in the investigations of DIBs.

\section{Families}

The term "families", concerning DIBs was used for the first time in the literature in relation to DIBs by Krełowski and Walker (1987). In high resolution and high SNR spectra it became clear that the strength ratio of the major DIBs 5780 and 5797 changes from object to object. It is interesting that the authors observed the same target in which M.L. Heger for the first time observed DIBs: HD24398 ( $\zeta$ Per); the 5780/5797 intensity ratio in this object is evidently different than in, say HD40111. A similar result was published soon after by Josafatsson and Snow (1987); the variable DIB strength ratio could be seen e.g. in HD30614 vs. HD38087. Such a highly variable ratio of the major DIB EWs clearly suggested that they are not of common origin but belong to different "families" considered as sets of features sharing the same carriers.

The above mentioned result was, however, known earlier. This is the part of history that I can recall as one of the oldest researchers in the DIB branch. I have seen the variable 5780/5797 ratio in February 1983 in the spectra acquired at the ESO CAT/CES system by Bengt Westerlund - then the director of Uppsala Observatory. The variable ratio was so astonishing at the time (the above mentioned spectra were among the first digitally recorded ones) that we didn't dare to publish them officially before the whole sample was re-observed and compared to other results. This is why this result was published as late as 1988 (Krełowski \& Westerlund 1988). The only way in which it was presented to the community was an oral communication during the European Regional Meeting held in Florence in December 1983.

However, something can be traced in the contemporary literature which is rooted in the above mentioned project. The two objects in which we observed the variable ratio were HD 147165 ( $\sigma \mathrm{Sco})$ and $\mathrm{HD} 149757$ ( $\zeta \mathrm{Oph}$ ). The terms $\sigma$ and $\zeta$ type clouds, popular now, are rooted in that "ancient" project in which the variable strength ratio was observed for the first time. The objects were later observed several times and the $\sigma$ and $\zeta$ type clouds were connected not only to the $5780 / 5797$ ratio but also to the shapes of extinction curves (see Fitzpatrick \& Massa 2007) and to the relative abundances of simple interstellar radicals. Both DIBs and bands carried by simple molecules are depicted in Fig. 1, presenting the two archetypes of $\sigma$ and $\zeta$ type clouds.

Naturally the determination of the rest wavelengths of various DIBs is the first necessary step toward identification. Every object in the Universe moves slower or faster in relation to the observer. Thus to obtain laboratory wavelengths of unidentified interstellar features it is necessary to move the wavelength scale to the rest wavelength frame for the interstellar medium. In fact, a vast majority of reddened OB stars are observed through more than one cloud. This causes Doppler splitting of interstellar lines and makes this task very difficult. One can use for it only certain, carefully selected targets, where the 


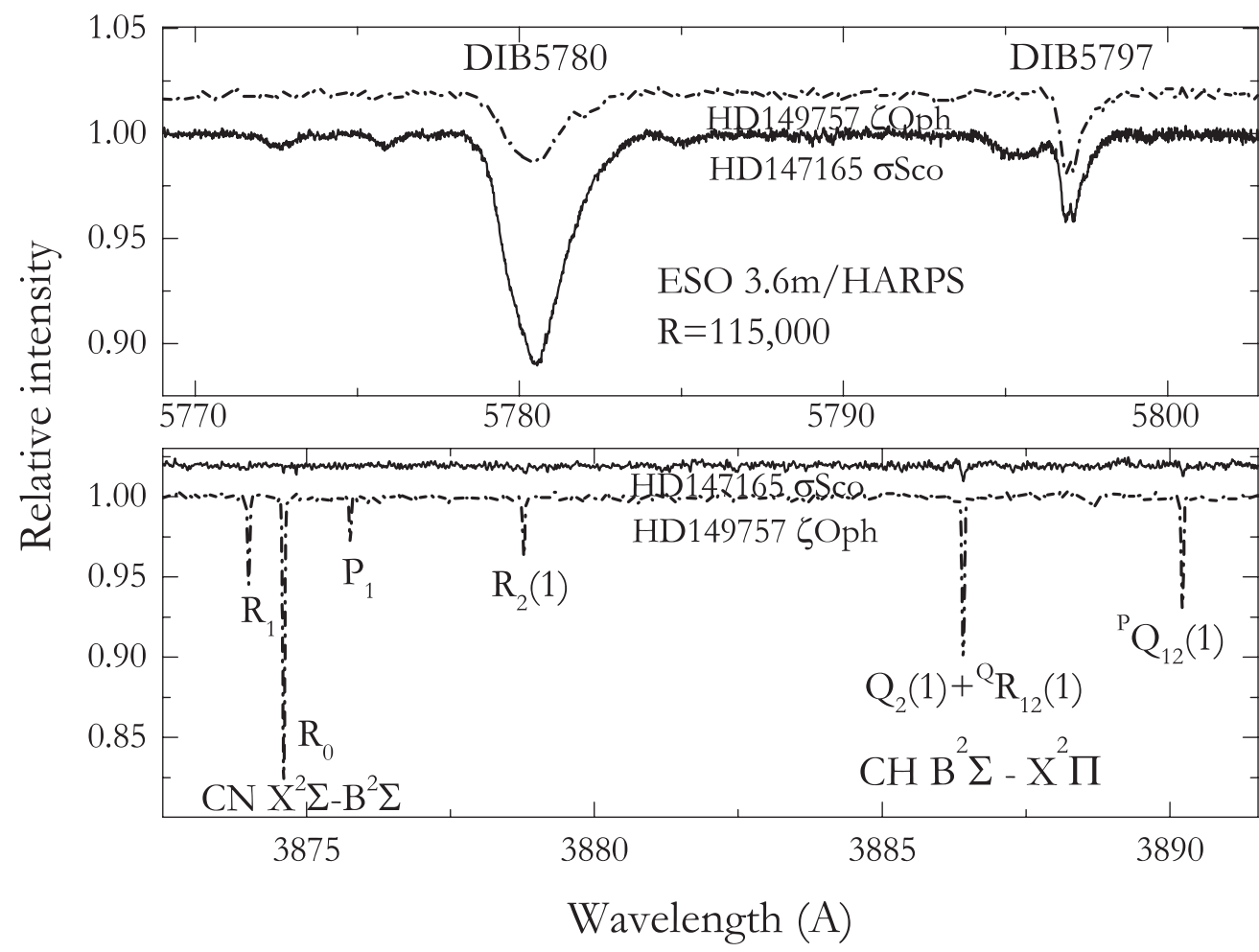

Figure 1. Major DIBs (upper panel) and the bands of simple interstellar radicals, observed in the spectra of HD 147165 and HD 149757 - the archetypes of $\sigma$ and $\zeta$ type clouds. Note the opposite ordering of the objects in the two panels!

Doppler splitting is not observed in atomic and/or molecular lines, being usually much narrower than DIBs. If we observe coinciding radial velocities in all identified lines and bands in a given target then it is reasonable to assume that it is observed through just one cloud. Such objects, very scarce in fact, are to be used to determine rest wavelengths of DIBs - the values necessary for the carriers' identification.

The problem of the intrinsic wavelengths of DIBs may, however, be more complex than it seemed earlier. Figure 2 compares the spectra of two stars that appear to be seen through single clouds. This is inferred from the fact that the radial velocities of all identified interstellar spectral features observed toward these stars are identical. The latter statement is especially strong because of the spectrograph used: it is HARPS - the instrument which guarantees the most precise and accurate wavelength determinations among the major astrophysical spectrographs in the world. The spectra are normalized to the depth of the 5780 DIB. It is evident that the wavelengths of most of the DIBs, a strong 5797 and a couple of weak ones, coincide. It is, however, not the case of the strongest 5780, for which the DIB is apparently broadened and redshifted in the spectrum of HD 147889. The reason for this phenomenon remains, however, unknown. In any case, a possible conclusion is that if two DIBs always share the same radial velocities, then they may belong to the same "family" considered as the spectrum of a single carrier. This is a necessary condition for creating a "family", but not a sufficient one.

Diffuse bands, if observed in objects which shine through several interstellar clouds may be more or less Doppler split. This splitting is in most of cases hardly detectable because 


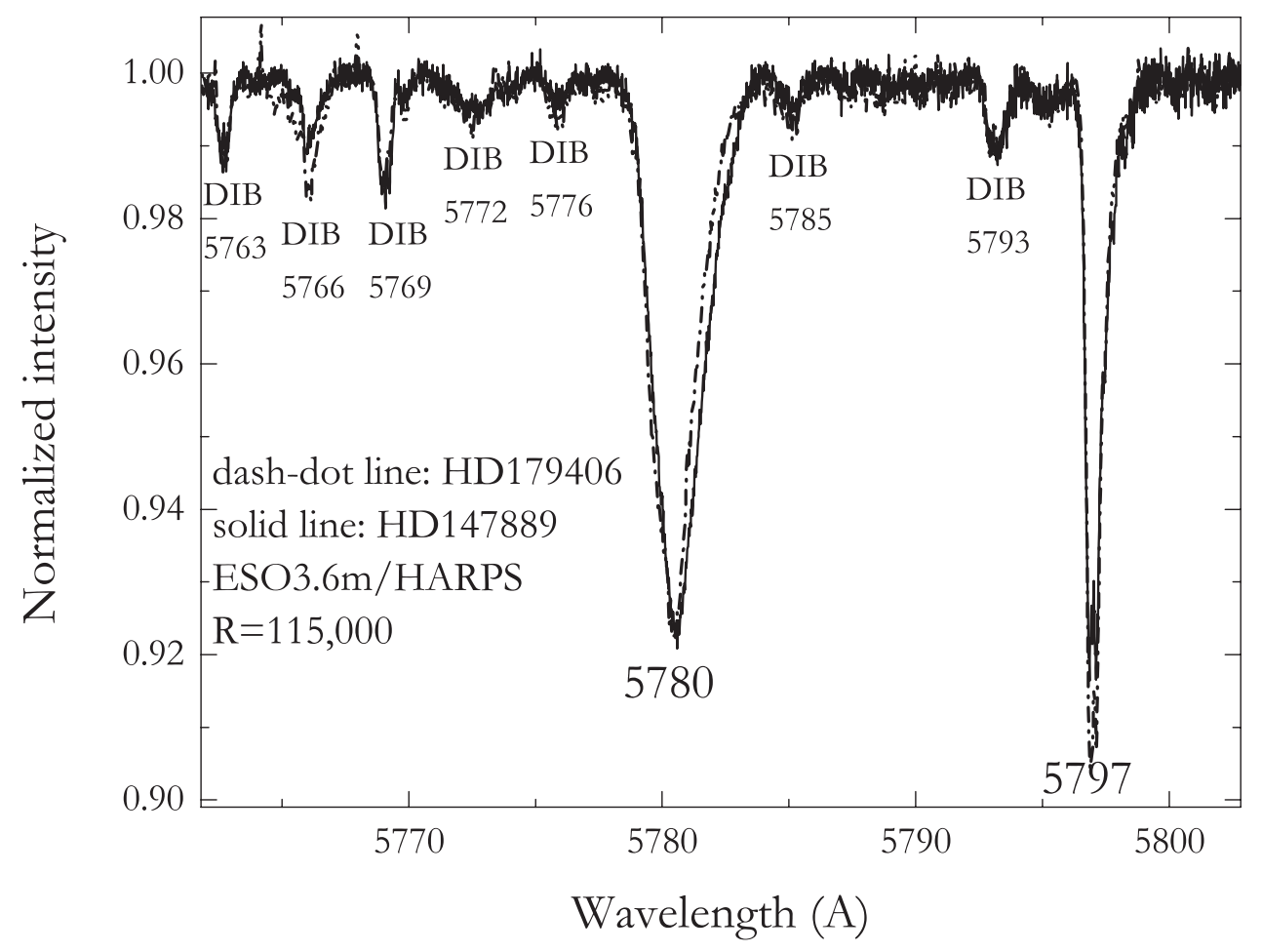

Figure 2. The HARPS spectra of HD 179406 and HD 147889 covering the range of major DIBs, normalized to the depth of 5780 . Note the broadening and redshift of the broad 5780 DIB.

the DIB profiles are relatively broad. In the cases of small relative radial velocities of the intervening clouds, one can observe only some modification of the DIB profiles (Krełowski \& Westerlund 1988). Usually also the substructures, discovered by Sarre et al. (1995) and seen clearly e.g. in Fig. 2, disappear. The Doppler structures in DIB profiles were discovered only in 1982 by Herbig \& Soderblom. They found the effect of radial velocities differing by $\sim 17 \mathrm{~km} / \mathrm{s}$ in the spectrum of HD 183143. A much stronger effect, found by Weselak et al. (2010), is demonstrated below in Fig. 3.

With the growing SNR of digitally recorded spectra, the number of known DIBs started growing very rapidly. The echelle spectrographs enabled the simultaneous use both of high resolution and broad wavelength range. This technological novelty resulted in broad surveys of DIBs - especially weak, previously unknown features. Jenniskens \& Désert (1994) reported 64 certain new DIBs and 69 possible ones. A similar survey by Krełowski, Sneden \& Hiltgen (1995) showed 62 additional features in a limited wavelength range. The broad survey by Galazutdinov et al. (2000) presented a list of 271 DIBs. This number has grown today to 414 listed features (Hobbs et al. 2009) found in the spectrum of HD 183143 - the heavily reddened object already used by Herbig (1975) to illustrate different DIB profiles. The survey presenting the most precise rest wavelengths of DIBs (Bondar 2012) lists 357 features observed in more than one object each.

It is obvious that such a large number of individual features cannot be carried by just one substance. This makes it very likely that the idea of DIB families is required. It also 


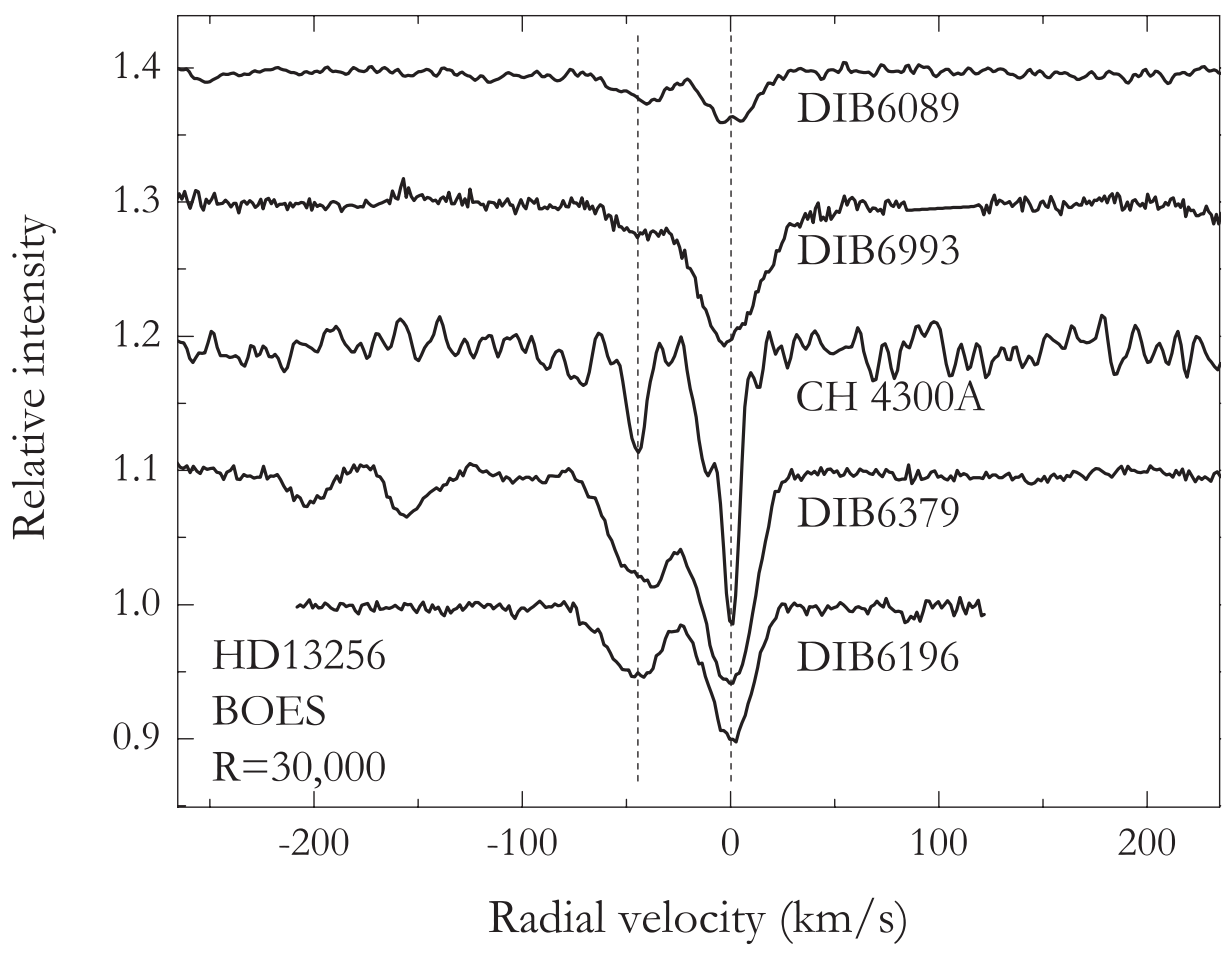

Figure 3. The evident Doppler splitting of four DIBs - the same as in $\mathrm{CH}$ interstellar line seen in the spectrum of the distant supergiant.

practically rules out dust grains as possible DIB carriers, since we must look for a great variety of carriers, making the molecular hypothesis more attractive. If DIBs are carried by some complex molecular species one can expect that each carrier may be revealed by one reasonably strong feature and several much weaker ones. Thus every "family" assumed as a spectrum of a single molecule should contain DIBs of various intensities. This makes deep surveys, based on very high SNR spectra, very important. It is very likely that among stronger DIBs the situation is: one DIB - one carrier; weak features added to possible "families" are thus of fundamental importance. Herbig (1988) was the first to point to a set of rather weak DIBs found between 6767 and $6862 \AA$. This set contains five or six DIBs which are nearly uniformly spaced at intervals of $35 \mathrm{~cm}^{-1}$, which may be characteristic for certain molecular carriers. Unfortunately, the carrier was not identified. Weselak et al. (2001) made a similar attempt to search for equally spaced "families" among stronger DIBs, with the same negative result. A similar set was found by A. Bondar (poster at this meeting); unfortunately the currently available laboratory data do not allow an identification.

It seems natural that the intensities of any two members of the same "family" should correlate rather tightly. This was the motivation for a few surveys aiming at determination of the correlation between different, usually quite strong DIBs. The first such survey (Moutou et al. 1999) found the pair that correlates almost perfectly: 6196 and 6614. The former is quite narrow, the latter - quite broad. Their EW ratio is nearly 4 . This tight correlation was confirmed later by other teams, e.g. McCall et al. (2010) - it is thus established beyond reasonable doubt. However, Galazutdinov et al. (2002) demonstrated 


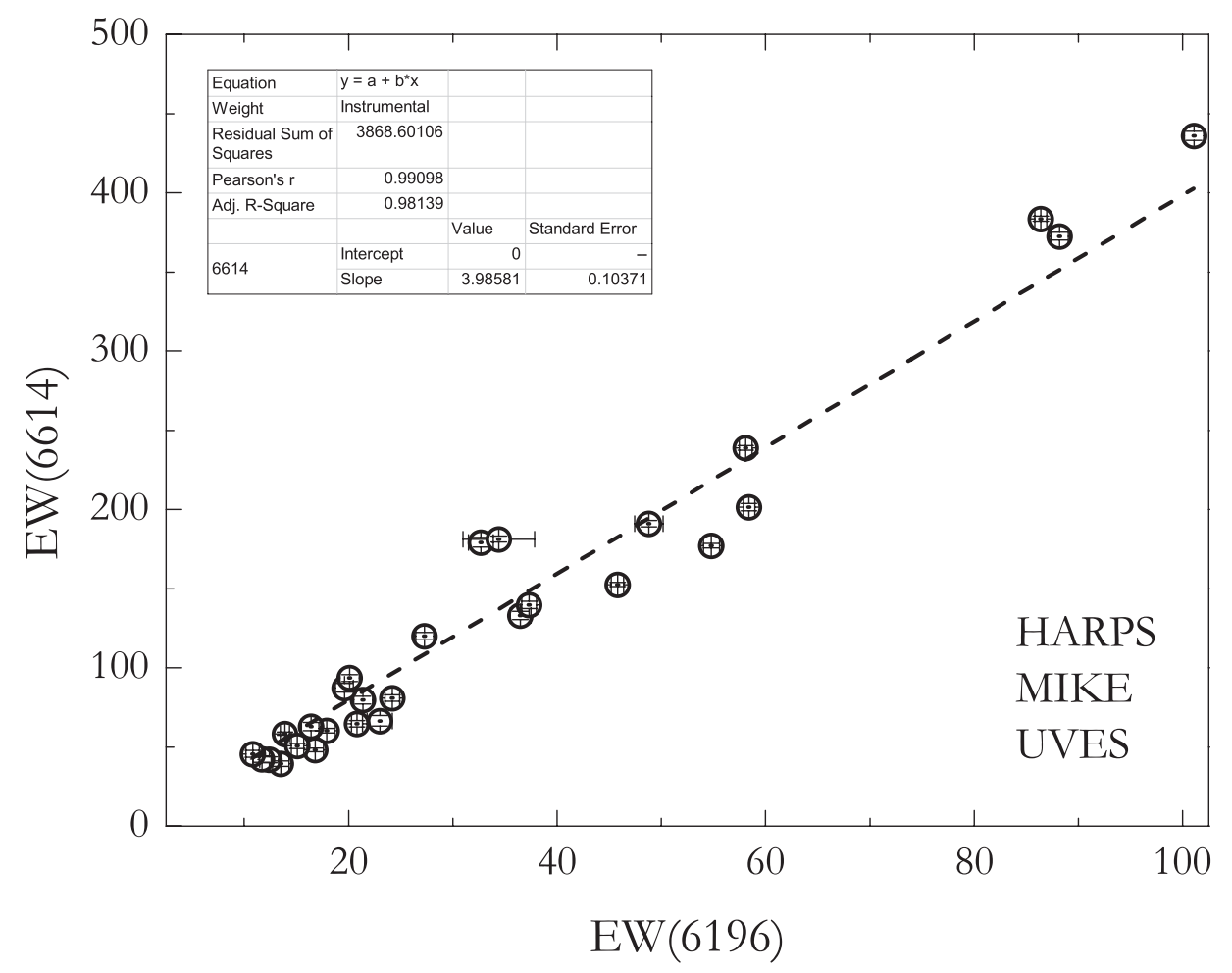

Figure 4. The tight correlation between EWs of the 6196 and 6614 DIBs based on spectra from major world spectrographs. Formal measurement errors mostly remain smaller than the symbol sizes. However, the scatter may result from problems with the continuum level setting.

that while the strengths of the two DIBs are well correlated, their FWHMs (full-width half-maxima) are not. Figure 4 presents the above mentioned tight correlation (very high correlation coefficient is shown) based on spectra from three major world spectrographs. This correlation may be considered as an argument for a common origin of the two DIBs. Once again it is a necessary but not a sufficient condition. Analyzing a spectrum of the undisputable hit of this Symposium, Herschel 36 (HD 164740), it is possible to find more spectacular evidence that the FWHMs of the two DIBs do not behave in unison (Fig. 5).

It is evident that in Herschel 36, the 6614 DIB has an extended red wing; the latter is barely seen in the case of 6196 . This creates serious doubt on whether the two features belong to the same "family", despite the tight correlation of their strengths shown above.

Specific objects can play a very important role in the process of DIB identification. "Peculiar" lines of sight are likely to intersect just single clouds where physical parameters can be defined clearly while "typical" objects are likely to be ill-defined averages of several different clouds. Some objects are known because DIB intensities observed in their spectra are very weak for their measured E(B-V). One such object is HD62542, mentioned by Snow et al. (2002) as almost free of DIBs when $\mathrm{E}(\mathrm{B}-\mathrm{V})=0.35$. Let us compare this object with another one of nearly the same reddening. The latter is HD 154445 which, according to Fitzpatrick \& Massa (2007), seems to be a $\sigma$ type object, i.e. with a strong 2200 


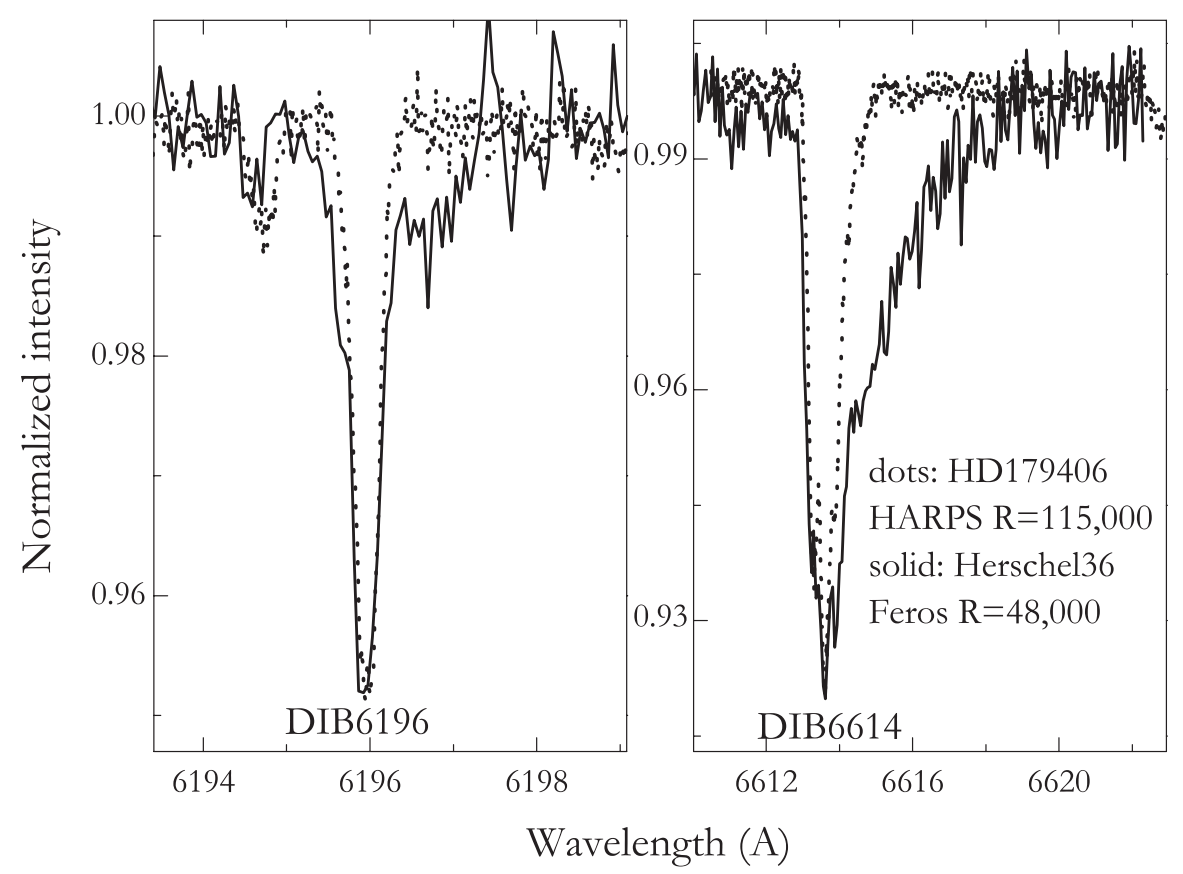

Figure 5. The 6196 and 6614 DIB profiles in HD 179406 and in Herschel 36. It is evident that the profiles behave in a different fashion. This may rule out their common origin.

extinction hump. HD62542 is rather a $\zeta$ type object with a steep far-UV part of the extinction curve and a very weak extinction bump.

Figure 6 shows clearly that while both major DIBs are very weak in HD 62542 the $\mathrm{CH}$ radical seems to be twice as abundant as in HD 154445. However, a real difference is seen in the $\mathrm{CN}$ radical! In HD 62542 the $\mathrm{R}(0)$ line of the ${ }^{13} \mathrm{CN}$ band is as strong as its ${ }^{12} \mathrm{CN}$ counterpart in HD 154445 ! A typical ${ }^{12} \mathrm{C} /{ }^{13} \mathrm{C}$ ratio is about 70 . Naturally, the band in HD 62542 is saturated, but in any case, the abundance of $\mathrm{CN}$ is at least an order of magnitude higher in this object of the same E(B-V). Apparently we face three players in translucent interstellar clouds: dust grains, simple radicals and carriers of DIBs. It should be emphasized that overabundance of simple species (like CN) may follow either the catalytic role of grain surfaces or the shattering of more complex species. A solution is now impossible.

Galazutdinov et al. (2006) discovered blueshifts of some DIBs in the spectrum of AE Aur (HD34078). The effect was small but evident. It was found in several objects of the Sco OB1 association by Galazutdinov et al. (2008), being of the same size as in AE Aur. The latter paper also demonstrated that the effect does not concern all DIBs some of them remain at the same intrinsic wavelengths. The discovery was based on spectra from different instruments: BOAO/BOES (Korea), Terskol/Maestro (Russia), ESO 2.2m/Feros and ESO 3.6m/HARPS. Because identical instrumental effects in all these spectrographs are unlikely, the effect, though small, must be considered to be real. Figure 7 demonstrates this using newly acquired spectra from the Magellan/MIKE system. It is evident that the above mentioned shift of the 6196 DIB is well seen between AE Aur and HD73882; on the other hand the effect does not exist in the case of the weak 4964 DIB. 


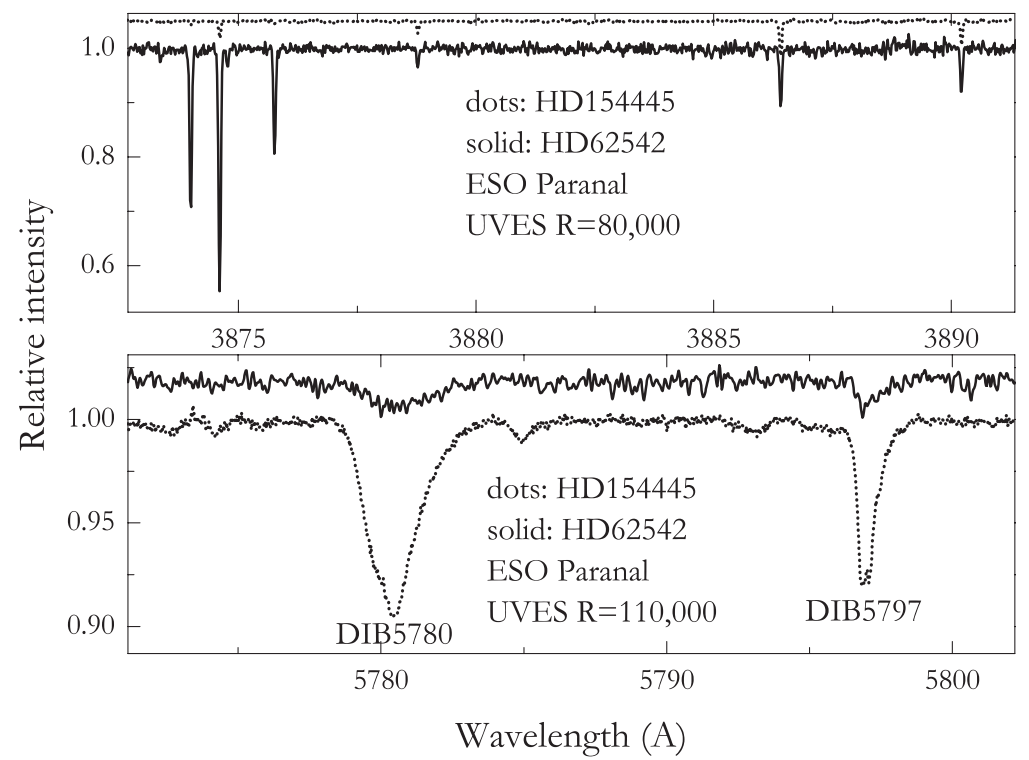

Figure 6. Molecular bands and major DIBs in two spectra with the same E(B-V) but evidently different extinction curves. For molecular features see Fig. 1. Note that the spectra are ordered differently in the two panels!

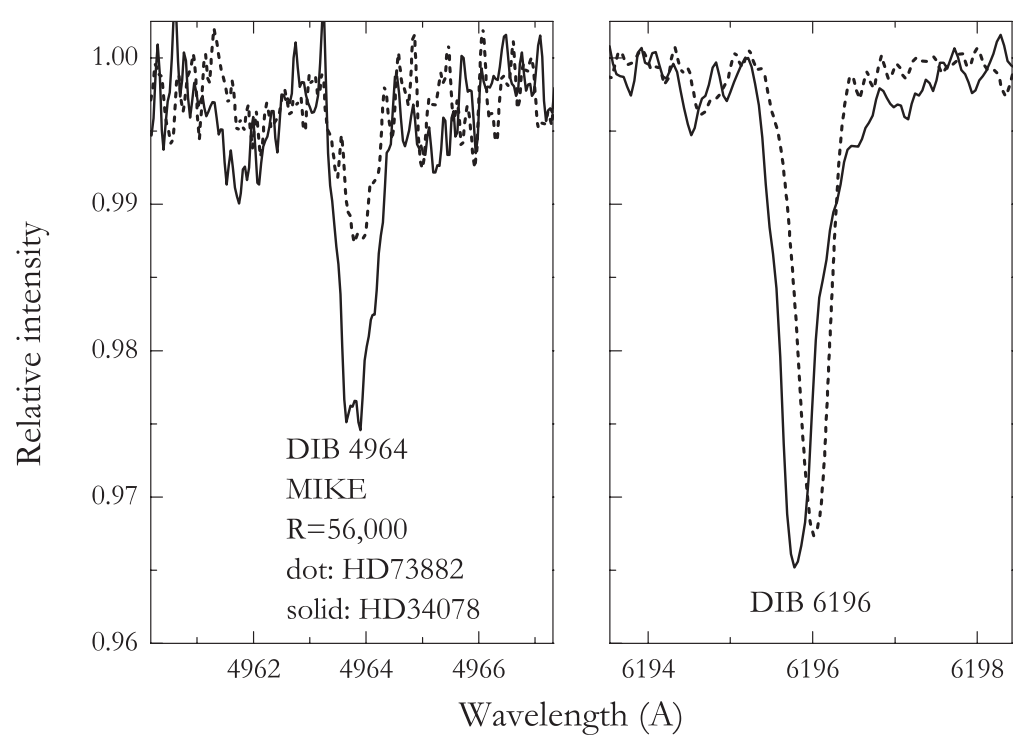

Figure 7. The 6196 DIB evidently blue-shifted in the spectrum of AE Aur when compared to that of HD73882. Note the lack of this effect in the case of 4964 DIB.

Thorburn et al. (2003) pointed to a set of rather weak DIBs whose intensities are to the interstellar $\mathrm{C}_{2}$ column density. This relation may be another indicator of the membership of a single "family". It is interesting that all these " $\mathrm{C}_{2}$ " DIBs are weak and occupy rather blue than red part of spectrum. Figure 8 demonstrates the correlations between two " $\mathrm{C}_{2}$ " DIBs and two other much stronger features. Naturally, the equivalent widths of the 6196 

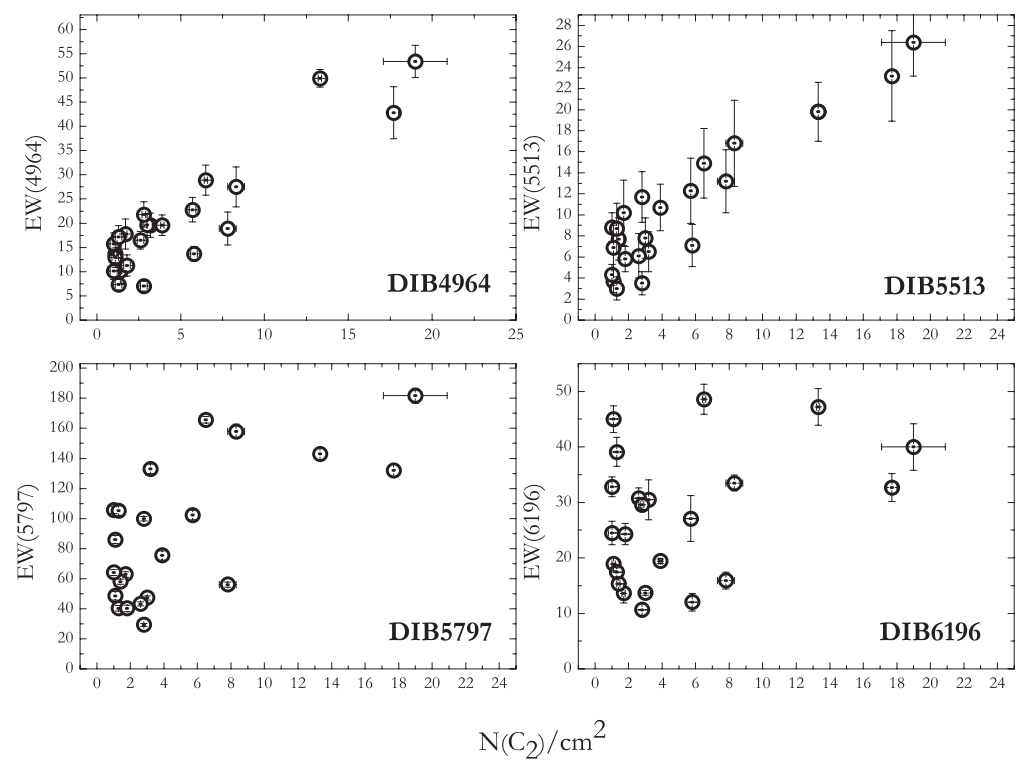

Figure 8. The " $\mathrm{C}_{2}$ " DIBs 4964 and 5513 correlated with the $\mathrm{C}_{2}$ column density. Such a correlation does not exist in the cases of much stronger 6196 and 5797 features.

and 5797 DIBs can be measured with a better precision because they are much stronger than 4964 or 5513 . However, they do not correlate with the $\mathrm{C}_{2}$ column density while the weaker DIBs do. The 5797 and 6196 show the blue-shifts in AE Aur and Sco OB1 objects while the weaker DIBs do not. It remains, however, undecided whether all the " $\mathrm{C}_{2}$ " DIBs are of the same origin or it is just a loose correlation. It is very difficult to improve the precision of " $\mathrm{C}_{2}$ " DIB measurements because all these features are quite weak, so the SNR inside their profiles remains low. High precision determinations of the $\mathrm{C}_{2}$ column density are also not easy. The molecule is homonuclear and thus the absorption splits into many transitions (depending on rotational temperature). Precise measurements of all these lines require a very high SNR, which is not always available.

\section{Discussion}

The idea of DIB "families" - sets of features sharing the same carriers seems natural. However, all the attempts to significantly establish such "families" seemingly fail. We observe certain similarities but the very rich spectrum of DIBs still remains to be a puzzle - it remains the longest standing unsolved problem in all of spectroscopy. As demonstrated above, all the ideas attributing sets of DIBs to any "family" face serious problems:

- correlations between DIB strengths do not guarantee that profiles of any two features always behave in the same fashion (Figs. 4 and 5);

- relations between simple molecular species and DIBs do not lead to any clear-cut results (Figs. 1 and 6); the third player is proved to be dust but the latter's role is not clear at the moment;

- relations between DIBs and $\mathrm{C}_{2}$ do not lead to any clear conclusion (Fig. 8). For some reason they concern only a handful of weak DIBs. The scarcity of precise $\mathrm{C}_{2}$ column density measurements play an obvious role here; more high resolution and SNR spectra are clearly necessary; 


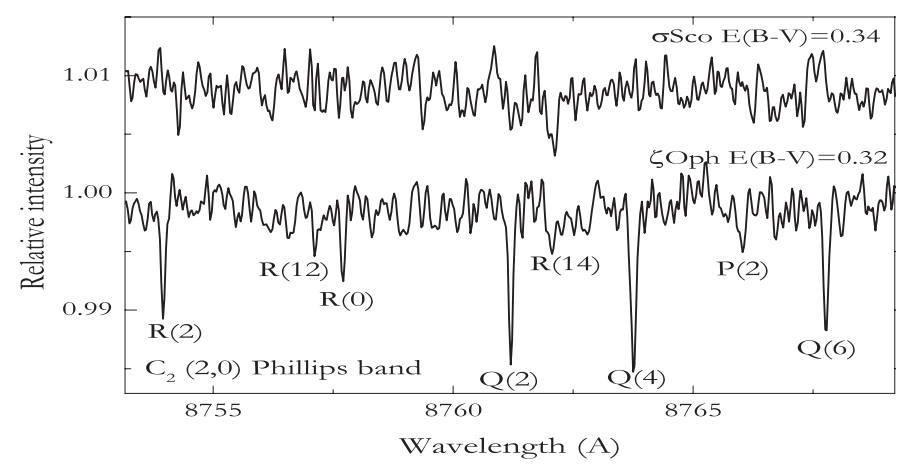

Figure 9. The $\mathrm{C}_{2}$ Phillips $(2,0)$ band in HD 147165 and HD 149757. The $\mathrm{C}_{2}$ column density in $\sigma$ Sco is below the level of detection. Spectra from Paranal/UVES R=110,000.

- the observed (Figs. 2 and 7) blue- and red- shifts (Krełowski \& Greenberg 1999) of some DIBs prevent a reliable division of DIBs between well-defined "families". The objects in which we observe such shifts are very scarce and thus any statistically wellgrounded conclusions are not possible.

The most promising are the $\mathrm{C}_{2}$ DIBs because they share two important properties: their intensities are related to that of $\mathrm{C}_{2}$ and they do not share blue-shifts with several much stronger features. Unfortunately they are all rather weak bands and thus the SNR of their profiles is low. We cannot measure their EWs with a sufficient precision. Moreover, $\mathrm{C}_{2}$ is not easy - see Fig. 9. The $\mathrm{C}_{2}$ bands typically contain many weak lines; moreover, in some objects (of the $\sigma$ type) this molecule falls below the detection threshold.

In general, investigations of the physics of the interstellar medium suffer because of the poor statistical properties of the observed samples of objects. In the vast majority of cases we observe some OB reddened stars through several translucent clouds and the visible picture is an ill-defined average involving several, probably different, environments. This fact makes all "peculiar" cases very important. They are most likely single cloud cases, i.e. they represent mostly homogeneous environments which may allow a physical interpretation. Such objects are, however, very scarce and thus we need many more observations so that single cloud cases could be selected and observed with very high resolution and SNR. This conference brought to the community a very interesting object Herschel 36 (HD 164740). This is the only observed object in which we can see transitions from rotationally excited levels of $\mathrm{CH}$ and $\mathrm{CH}^{+}$molecules. Let us emphasize that polar molecules are excited only in very specific environments. Despite the above mentioned object there are two others where the CN molecule is evidently excited: HD34078 and Tr16 112 (Krełowski, Galazutdinov \& Gnaciński 2012). Unfortunately in each of these three objects DIBs behave in a different fashion: in Herschel 36 - see Fig. 4, in AE Aur most of them demonstrate a blue-shift, in Tr16 112 they are just a bit broadened. No obvious conclusions are thus possible. Hence, after nearly 40 years of being involved in the DIB investigations I am just going to pay a tribute to Mr. Murphy, whose famous law is never disobeyed!

\section{Acknowledgements}

I acknowledge the financial support of the Polish National Science Center during the period 2011 - 2013 (grant N N203 582040) and the very valuable remarks of Dr. B. Roukema. 


\section{References}

Bondar, A. 2012, MNRAS, 423, 725

Fitzpatrick, E. L. \& Massa, D. 2007, ApJ, 663, 320

Galazutdinov, G. A., Musaev, F. A., Krełowski, J., \& Walker, G. A. H. 2000, PASP, 112, 648

Galazutdinov, G. A., Moutou, C., Musaev, F. A., \& Krełowski, J. 2002, AA, 384, 215

Galazutdinov, G. A., Manicó, G., \& Krełowski, J. 2006, MNRAS, 366, 1075

Galazutdinov, G. A., Locurto, G., Han, Inwoo, \& Krełowski, J. 2008, PASP, 120, 178

Heger, M. L. 1922, Lick Obs. Bull. 10, 146

Herbig, G. H. 1975, ApJ, 196, 129

Herbig, G. H. 1988, ApJ, 331, 999

Herbig, G. H. \& Soderblom, D. R. 1982, ApJ, 252, 610

Hobbs, L. M., York, D. G., Thorburn, J. A., Snow, T. P., Bishof, M., Friedman, S. D., McCall, B. J., Oka, T., Rachford, B., Sonnentrucker, P., \& Welty, D. E. 2007, ApJ, 705, 32

McCall, B. J. \& Griffin, R. E. 2013, Proc. R. Soc. A, 469, 20120604

Merrill, P. W. \& Wilson, O. C. 1938, ApJ, 87, 9

Jenniskens, P. \& Desert, F.-X. 1994, AAS, 106, 39

Josafatsson, K. \& Snow, T. P. 1987, ApJ, 319, 436

Krełowski, J. \& Walker, G. A. H. 1987, ApJ, 312, 860

Krełowski, J. \& Westerlund, B. E. 1988, AA, 190, 339

Krełowski, J., Sneden, C., \& Hiltgen, D. 1995, PSS 43, 1195

Krełowski, J. \& Greenberg, J. M. 1999, AA, 346, 199

Krełowski, J., Galazutdinov, G. A., \& Gnaciński, P. 2012, AN 333, 627

McCall, B. J., Drosback, M. M., Thorburn, J. A., York, D. G., Friedman, S. D., Hobbs, L. M., Rachford, B. L., Snow, T. P., Sonnentrucker, P., \& Welty, D. E. 2010, ApJ, 708, 1628

Moutou, C., Krełowski, J., D'Hendecourt, L., \& Jamroszczak, J. 1999, AA, 351, 680

Sarre, P. J., Miles, J. R., Kerr, T. H., Hibbins, R. E., Fossey, S. J., \& Somerville, W. B. 1995, MNRAS, 277, L41

Snow, Theodore P., Welty, Daniel E., Thorburn, J., Hobbs, L. M., McCall, B. J., Sonnentrucker, P., \& York, D. G. 2002, ApJ, 573, 670

Thorburn, J. A., Hobbs, L. M., McCall, B. J., Oka, T., Welty, D. E., Friedman, S. D., Snow, T. P., Sonnentrucker, P., \& York, D. G. 2003, ApJ, 584, 339

Weselak, T., Fulara, J., Schmidt, M. R.,\& Krełowski, J. 2001, AA, 377, 677

Weselak, T., Galazutdinov, G. A., Han, Inwoo, \& Krełowski, J. 2010, MNRAS, 401, 1308 